PROCEEDINGS OF THE

AMERICAN MATHEMATICAL SOCIETY

Volume 128, Number 11, Pages 3421-3423

S 0002-9939(00)05438-1

Article electronically published on May 18, 2000

\title{
ON THE SET OF POINTS WITH A DENSE ORBIT
}

\author{
NILSON C. BERNARDES, JR. \\ (Communicated by Alan Dow)
}

\begin{abstract}
Under certain conditions on the topological space $X$ we prove that for every continuous map $f: X \rightarrow X$ the set of all points with a dense orbit has empty interior in $X$. This result implies a negative answer to two problems proposed by M. Barge and J. Kennedy.
\end{abstract}

\section{INTRODUCTION}

Let $X$ be a topological space. Given a subset $A$ of $X$ we shall denote by $\mathrm{Cl}_{X}(A)$ and $\operatorname{Int}_{X}(A)$ the closure and the interior of $A$ in $X$, respectively. If $U, V$ are subsets of $X \times X$ and $a \in X$, we define

$$
U \circ V=\{(a, b) \in X \times X ;(a, c) \in U \text { and }(c, b) \in V \text { for some } c \in X\}
$$

and

$$
U(a)=\{b \in X ;(a, b) \in U\} .
$$

Moreover, if $f: X \rightarrow X$ is a continuous map, the orbit of a point $x \in X$ under $f$ is the set

$$
O_{f}(x)=\left\{f^{j}(x) ; j \geq 0\right\} .
$$

Finally, we shall denote by $D_{f}$ the set of all points $x \in X$ whose orbit $O_{f}(x)$ is dense in $X$.

M. Barge and J. Kennedy [1] proposed the following problems (cf. Problems 5 and 6 on page 641 of [1]):

- Let $\left\{p_{1}, p_{2}, \ldots, p_{n}\right\}$ be a set of $n \geq 2$ distinct points in the sphere $S^{2}$. Is there a homeomorphism of $S^{2}-\left\{p_{1}, p_{2}, \ldots, p_{n}\right\}$ such that every orbit of the homeomorphism is dense?

- Is there a homeomorphism of $\mathbf{R}^{n}, n \geq 3$, such that every orbit of the homeomorphism is dense?

Our goal is to answer both questions in the negative. More precisely, we shall see that if $X=S^{2}-\left\{p_{1}, p_{2}, \ldots, p_{n}\right\}$ or $X=\mathbf{R}^{n}(n \geq 1)$, then any continuous map $f: X \rightarrow X$ has the property that $\operatorname{Int}_{X}\left(D_{f}\right)=\emptyset$ (in particular, $D_{f} \neq X$ ). In fact, our results apply to a much larger class of spaces $X$ (cf. Theorem 1 and Corollary 6).

Received by the editors October 19, 1998 and, in revised form, January 22, 1999

2000 Mathematics Subject Classification. Primary 37B20, 54H20.

(C)2000 American Mathematical Society 


\section{MAin Results}

Theorem 1. Let $X$ be a locally compact Hausdorff space which is not compact and has no isolated points. Then, for any continuous map $f: X \rightarrow X$, we have $\operatorname{Int}_{X}\left(D_{f}\right)=\emptyset$.

Proof. Let $Y$ be the one-point compactification of $X$ and let $\mathcal{U}$ be the set of all open symmetric vicinities of the unique uniform structure compatible with the topology of $Y$. Fix $z \in \mathrm{Cl}_{Y}(X)-X$ and suppose that $\operatorname{Int}_{X}\left(D_{f}\right) \neq \emptyset$. Then, there are $x_{0} \in X$ and $V \in \mathcal{U}$ such that

$$
\mathrm{Cl}_{Y}\left(V\left(x_{0}\right)\right) \subset D_{f}
$$

For each $U \in \mathcal{U}$, choose an integer $r_{U} \geq 1$ such that $f^{r_{U}}\left(x_{0}\right) \in U(z)$. Let $\alpha_{U} \leq r_{U}$ be the greatest integer such that $f^{\alpha_{U}}\left(x_{0}\right) \in V\left(x_{0}\right)$ and let $\beta_{U}>r_{U}$ be the smallest integer such that $f^{\beta_{U}}\left(x_{0}\right) \in V\left(x_{0}\right)$. Put $y_{U}=f^{\alpha_{U}}\left(x_{0}\right)$. Then we have the following properties:

$$
\begin{gathered}
y_{U}, f^{\beta_{U}-\alpha_{U}}\left(y_{U}\right) \in V\left(x_{0}\right), \\
f\left(y_{U}\right), f^{2}\left(y_{U}\right), \ldots, f^{\beta_{U}-\alpha_{U}-1}\left(y_{U}\right) \notin V\left(x_{0}\right)
\end{gathered}
$$

and

$$
f^{r_{U}-\alpha_{U}}\left(y_{U}\right) \in U(z)
$$

Let $y_{0} \in Y$ be a cluster point of the net $\left(y_{U}\right)_{U \in \mathcal{U}}$. Since $y_{0} \in \mathrm{Cl}_{Y}\left(V\left(x_{0}\right)\right) \subset D_{f}$, there is a smallest integer $M \geq 1$ such that

$$
f^{M}\left(y_{0}\right) \in V\left(x_{0}\right) .
$$

Let $Z \in \mathcal{U}$ and $V^{\prime} \in \mathcal{U}$ be such that

$$
\left(f^{M}\left(y_{0}\right), x_{0}\right) \in V^{\prime} \text { and } Z \circ V^{\prime} \subset V .
$$

For each $U \in \mathcal{U}$, let $N_{U}$ be a neighborhood of $y_{0}$ in $Y$ such that

$$
\left(f^{n}(y), f^{n}\left(y_{0}\right)\right) \in Z \cap U \text { for } n=0, \ldots, M \text {, whenever } y \in X \cap N_{U} .
$$

Since $y_{0}$ is a cluster point of $\left(y_{U}\right)_{U \in \mathcal{U}}$, there is a $W_{U} \in \mathcal{U}, W_{U} \subset U$, such that

$$
y_{W_{U}} \in N_{U} \text {. }
$$

Thus, $f^{M}\left(y_{W_{U}}\right) \in V\left(x_{0}\right)$. Since $\beta_{W_{U}}-\alpha_{W_{U}} \leq M$, we obtain

$$
\left(f^{r_{W_{U}}-\alpha_{W_{U}}}\left(y_{0}\right), z\right) \in U \circ U \text {. }
$$

Therefore, $z \in \mathrm{Cl}_{Y}\left(\left\{y_{0}, f\left(y_{0}\right), \ldots, f^{M}\left(y_{0}\right)\right\}\right)$, and so

$$
z=f^{j}\left(y_{0}\right) \text { for some } 0 \leq j \leq M \text {. }
$$

But this is a contradiction, since $z \notin X$.

As an immediate consequence of Theorem 1 we obtain the following negative answers to the two problems mentioned in the introduction:

Corollary 2. For any integer $n \geq 1$ and any continuous map $f: \mathbf{R}^{n} \rightarrow \mathbf{R}^{n}$, we have $\operatorname{Int}_{\mathbf{R}^{n}}\left(D_{f}\right)=\emptyset$.

Corollary 3. Let $X=S^{2}-\left\{p_{1}, \ldots, p_{n}\right\}$, where $p_{1}, \ldots, p_{n}(n \geq 1)$ are distinct points of $S^{2}$. Then, for any continuous map $f: X \rightarrow X$, we have $\operatorname{Int}_{X}\left(D_{f}\right)=\emptyset$. 
Remark 4. The conclusion of Corollary 2 is not true if we consider infinite-dimensional Banach spaces $X$ in place of $\mathbf{R}^{n}$. Indeed, in certain infinite-dimensional Banach spaces $X$, Read [2] proved the existence of a continuous map $f: X \rightarrow X$ such that $D_{f}=X-\{0\}$ (his map is even linear). This also shows that we cannot omit the local compactness hypothesis in Theorem 1 .

\section{Further RESUlts}

The argument used in the proof of Theorem 1 can also be applied to establish the following:

Theorem 5. Let $X$ be a compact Hausdorff space without isolated points. Then, for any continuous map $f: X \rightarrow X$, either $D_{f}=X$ or $\operatorname{Int}_{X}\left(D_{f}\right)=\emptyset$.

Proof. Suppose $D_{f} \neq X$ and $\operatorname{Int}_{X}\left(D_{f}\right) \neq \emptyset$. Fix an $a \in X-D_{f}$. By arguing as in the proof of Theorem 1 (with $z=a$ ), we conclude that for some $y_{0} \in D_{f}$ and some $j \geq 0$,

$$
f^{j}\left(y_{0}\right)=z=a .
$$

But this is a contradiction, since $y_{0} \in D_{f}$ and $a \notin D_{f}$.

Corollary 6. Let $X$ be a compact convex set in a Hausdorff locally convex space and assume that $X$ is not a singleton. Then, for any continuous map $f: X \rightarrow X$, we have $\operatorname{Int}_{X}\left(D_{f}\right)=\emptyset$.

Proof. By the Schauder-Tychonoff fixed point theorem, $f$ has a fixed point in $X$. So, we cannot have $D_{f}=X$.

Remark 7. (a) The possibility $D_{f}=X$ can happen in Theorem 5 For instance, let $X$ be the unit circle and let $f: X \rightarrow X$ be a rotation by an irrational number.

(b) We cannot omit the hypothesis that $X$ has no isolated points in Theorem 5 For instance, consider $X=\{0,1\}$ and define $f: X \rightarrow X$ by $f(0)=0$ and $f(1)=0$. Then $D_{f}=\{1\}$, which is not $X$ and does not have empty interior.

(c) In view of Read's example [2], we cannot omit the compactness hypothesis in Theorem 5 ,

\section{REFERENCES}

[1] M. Barge and J. Kennedy, Continuum theory and topological dynamics. In Open Problems in Topology, J. van Mill and G. M. Reed, editors, pages 633-644. Elsevier Science Publishers B. V. (North-Holland), 1990. CMP 91:03

[2] C. J. Read, The invariant subspace problem for a class of Banach spaces, 2: Hypercyclic operators, Israel J. Math. 63 (1988), 1-40. MR 90b:47013

Instituto de Matemática, Universidade Federal Fluminense, Rua Mário Santos Braga S/N, Niterói, RJ, 24020-140, BrasiL

E-mail address: ganncbj@vm.uff.br 